\title{
Exposure and Loss of Environmental Enrichment Mediates Ethanol Consumption in Adolescent Female Rats
}

\author{
Natalie Lipari*a, Max Baron ${ }^{b}$ \& Joshua A. Peck ${ }^{a}$ \\ ${ }^{a}$ Psychology Department, State University of New York at Cortland, Cortland, NY \\ ${ }^{\circ}$ Psychology Department, University of Michigan, Ann Harbor, MI \\ https:/ / doi.org/10.33697/ ajur.2019.032 \\ Students:natalie.lipari@cortland.edu*,maxbaron@umich.edu \\ Mentors:joshua.peck@cortland.edu
}

\begin{abstract}
Alcohol use among adolescent females has significantly increased in the United States with young women drinking alcohol at the same rate as young men. One potential treatment strategy that could help sustain alcohol abstinence is Environmental Enrichment (EE). Environmental enrichment is a process concerning the stimulation of the brain by one's physical and social surrounding, which promotes non-drug reinforcement alternatives (e.g. voluntary exercise) supporting drug abstinence. Thus, the primary focus of this study was to investigate the effect of EE on ethanol (ETOH) abstinence in adolescent female rats. All adolescent female rats, starting on postnatal day 30, had 24 -h access to $2 \%$, then $4 \%$, and then $6 \%$ ethanol concentrations. At the end of the four weeks, the environmental conditions were switched $(\mathrm{EE} \rightarrow \mathrm{NEE}$ and $\mathrm{NEE} \rightarrow \mathrm{EE})$ and the $6 \%$ ethanol measure was repeated. We found that EE significantly reduced ethanol consumption for adolescent female rats compared to controls. Further, the removal of EE opportunities resulted in a significant increase in ethanol consumption. Collectively, the results suggest that access to enriched life conditions are important in facilitating alcohol abstinence in adolescent female rats.
\end{abstract}

\section{KEYWORDS}

Adolescent Females; Alcohol Consumption; Environmental Enrichment; Alcohol Use Disorder; Treatment Strategy; Alcohol Abstinence; Ethanol; Adolescent Female Rats

\section{INTRODUCTION}

Alcohol use among young women has significantly increased in the United States within recent years. Young women are now drinking alcohol at similar rates as young men, suggesting that differences in consumption of alcohol for males and females has dramatically narrowed. ${ }^{1}$ For example, thirty-seven percent of ninth grade girls_-averaging at about 14 years old — report drinking in the past month, surpassing the percentage rate reported for ninth grade boys. ${ }^{2}$ Unfortunately, the increased use of alcohol in young women can set the stage for development of an alcohol use disorder (AUD) later in life. ${ }^{3,4}$ According to the National Institute of Health, AUD is a chronic relapsing brain disease characterized by compulsive alcohol use, loss of control over alcohol intake, and a negative emotional state when not using. ${ }^{2}$ Given the recent upward trend of AUDs among adolescent females, it is important that substance abuse animal research examine possible treatment strategies that may be effective for treating adolescent female alcohol abuse.

One promising treatment strategy that has shown to support drug abstinence is environmental enrichment. Environmental Enrichment (EE) can be defined as the non-contingent delivery of alternative non-drug rewards such as food, social interaction, novelty objects and voluntary physical activity either in the presence of drug (concurrent) or in the absence of drug (nonconcurrent). 5-12 Access to nondrug alternatives can impede or prevent acquisition and decrease drug-maintained responding. ${ }^{13,14}$ For example, given the choice between drug and other types of rewards (e.g., toys or social interaction) they will typically prefer the alternative rewards over drug.$^{5,6,13-17}$ Further, the removal of such non-drug alternatives may also result in increased drug taking. ${ }^{18,19}$

To date, research has provided promising evidence that EE may indeed support drug abstinence in male animal populations. Access to EE can prevent the acquisition of drug taking and decrease drug responding in male rats. ${ }^{6,13}$ For example, male animal studies have shown that EE reduces cocaine and heroin's reinforcing effects when concurrently available with the drug. ${ }^{10,14,15}$ However, there is a paucity of research concerning EE as a possible treatment strategy for substance abuse in female animal populations. To our knowledge, there have been no studies examining EE as a possible treatment method for alcohol consumption in adolescent female rats. Therefore, the current study examined a novel approach by implementing an EE treatment strategy that may help support alcohol abstinence in adolescent female rats. 
Our primary focus was to investigate the effects of exposure and loss of EE on ethanol (ETOH) consumption in adolescent female rats. During phase one, adolescent female rats were either placed in the EE or in standard non-enriched cages (NEE) with both groups having access to ETOH. For both groups, adolescent female rats were given 2\% ETOH for one week, then increased to $4 \%$ for another week and finished with $6 \%$ over the span of two weeks. At the end of the fourth week, we switched the groups (phase two) where female rats that were originally in the EE were now placed into the NEE and rats that were first in the NEE were given access to the enriched environment. We hypothesized that both groups of adolescent female rats would show significantly less ETOH consumption while in the EE condition, than in the NEE condition. If these results were to be observed then this would lend support for the implementation of EE as a strategy to facilitate alcohol abstinence in adolescent female rats.

\section{METHODS AND PROCEDURES}

Subjects

Twenty Sprague-Dawley adolescent female rats weighing between 300 and $350 \mathrm{~g}$ were used as subjects for both phases of the experiment. Estrous levels in free cycling females were not assessed. Each rat was housed in a climate-controlled environment ranging from 70.0-72.0 degrees Fahrenheit with constant access to water and Lab Rat Chow was provided during all phases of the experiment. Each rat was individually housed under a reversed 12-hour light: dark cycle (lights on at 19:00 h). This study complied with the guidelines of the National Institute of Health guide for the care and use of laboratory animals and was conducted in accordance with the SUNY Cortland's Institutional Animal Care and Use Committee ethics protocol (IACUC Protocol \#43).

\section{Environmental Enrichment}

Every female adolescent rat was provided a standard cage, $25 \times 20 \times 15 \mathrm{~cm}^{3}$ for the NEE and $60 \times 38 \times 20 \mathrm{~cm}^{3}$ for the EE (depending upon experimental phase). The EE contained running wheels, a 10-cm diameter tunnel, ladders, treats, and two additional objects that were rotated daily, such as a jingly ball, paper roll, and a dog chew toy. The components and procedure of rotating objects daily within enrichment cages, are similar to those used in other enrichment studies that have shown effects of the treatment. , $20-22^{2}$

\section{Procedure}

The procedure consisted of two four week phases. During phase 1, starting on postnatal day 30, adolescent female rats were assigned to either the EE ( $\mathrm{n}=10)$ or NEE $(\mathrm{n}=10)$ with a 14-oz drinking bottle containing an ascending series of ethanol concentrations placed on their home cages 24 hours per day (continuous access). Twenty female rats were exposed to different concentrations of ETOH by a fade-in procedure (Table 1). This procedure gradually introduces the rats to the ETOH solutions that provide a measure of volitional intake under restrictive conditions. ${ }^{21}$ This fade-in series started with $2 \%$ ETOH for the first seven days, followed by $4 \%$ for the next seven days and then $6 \%$ for 14 days. Bottles were weighed to the nearest $0.1 \mathrm{~g}$ at the same time daily. At the end of the fourth week, we switched the groups (phase 2) where female rats that were in the EE were now placed into the NEE (EE-NEE) and rats that were in the NEE were given access to the enriched environment (NEE-EE). During phase 2, all rats had continued access to $6 \%$ for 14 days.

\begin{tabular}{|l|c|c|c|}
\hline $\begin{array}{c}\text { Experimental Groups: } \\
(\text { EE } \rightarrow \text { NEE vs. NEE } \rightarrow \text { EE) }\end{array}$ & Week 1 & Week 2 & Weeks 3 \& 4 \\
\hline Phase 1: & $2 \%$ & $4 \%$ & $6 \%$ \\
Ethanol Exposure & 7 Days & 7 Days & 14 Days \\
Duration & & & \\
\hline Phase 2: Switched & $6 \%$ & $6 \%$ & N/A \\
Ethanol Exposure & 7 Days & 7 Days & \\
Duration & & \\
\hline
\end{tabular}

Table 1. Ascending series of ETOH concentrations administered during phases 1 and 2. 


\section{Statistical Analysis}

The dependent variable consisted of the amount of ethanol consumption $(6 \%)$ by the female rats across both phases. The independent variables were environmental history ( $\mathrm{NEE}$ or $\mathrm{EE})$ and groups $(\mathrm{EE} \rightarrow \mathrm{NEE} v s$. NEE $\rightarrow \mathrm{EE})$. A two-way ANOVA with group (between-subjects) and environmental history (repeated measures) was conducted comparing the mean $6 \%$ ETOH consumption amounts in the $\mathrm{EE}$ and $\mathrm{NEE}$ conditions for both $\mathrm{EE} \rightarrow \mathrm{NEE}$ and $\mathrm{NEE} \rightarrow \mathrm{EE}$ groups. Differences were considered significant at the $\mathrm{p}<0.05$ level. Standard error bars are indicated in Figure 1. All statistical analyses were conducted using IBM Statistical Package for Social Sciences (SPSS) 26 software.

\section{RESULTS}

A two-way ANOVA with group (between-subjects) and environmental history (repeated measures) as factors was conducted comparing the mean $6 \%$ ETOH consumption amounts in the EE and NEE conditions for both EE $\rightarrow \mathrm{NEE}$ and NEE $\rightarrow \mathrm{EE}$ groups. The $6 \%$ ETOH consumption over 14 days for the $\mathrm{EE} \rightarrow \mathrm{NEE}$ female adolescent rats during the NEE condition yielded a mean of $17.73 \pm 2.16$ grams $(\mathrm{g})$, while the $6 \%$ ETOH consumption over 14 days for the EE $\rightarrow$ NEE group during the EE condition led to a mean of $5.40 \pm 0.93(\mathrm{~g})$. The $6 \%$ ETOH consumption over 14 days for the NEE $\rightarrow$ EE rats during the NEE condition yielded a mean of $19.71 \pm 2.45(\mathrm{~g})$, while the $6 \%$ ETOH consumption over 14 days for this same group led to a mean of $7.54 \pm 1.23(\mathrm{~g})$. (Figure 1). For both $\mathrm{EE} \rightarrow \mathrm{NEE}$ and NEE $\rightarrow \mathrm{EE}$ groups, there was a significant effect of environmental history $[F(1,39)=16.59, p<.01]$. However, there were no significant effects for group $[F=3.59, p>.05]$ or group by environmental history interaction $[F=0.89, p>.05]$.

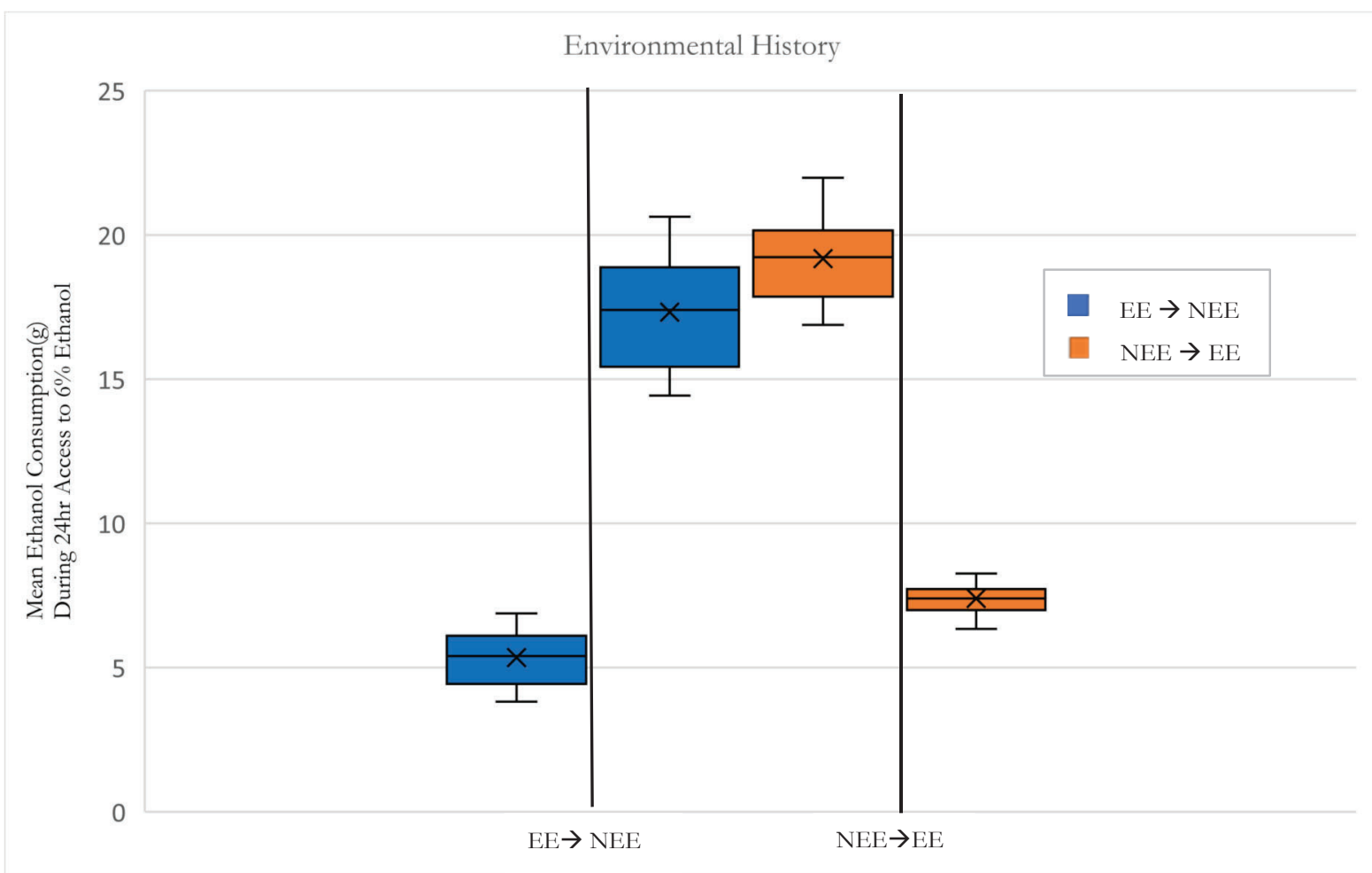

Figure 1: Mean $( \pm$ SEM) of $6 \%$ ETOH consumed during both phases for the $\mathrm{EE} \rightarrow \mathrm{NEE}$ and $\mathrm{NEE} \rightarrow \mathrm{EE}$ groups. $\mathrm{N}=10$ for each group

\section{DISCUSSION}

The current study examined a possible treatment method for supporting alcohol abstinence in adolescent female rats. We found that EE exposure led to significantly less consumption of ethanol for both female groups, while loss of EE led to a significant increase in ethanol intake. The results suggest that having access to EE opportunities while alcohol is readily available may be an effective treatment strategy for promoting decreased ethanol consumption in adolescent female rats. A possible explanation for this finding is that the introduction of rewarding stimulation experienced in the enriched environment might have reduced the reinforcing effects of the ethanol through a behavioral contrast mechanism. Behavioral contrast refers to a change in the rate of reinforcement on one component of a concurrent schedule produces an opposite change in the rate of response on another component creating an inverse relationship. ${ }^{23-25}$ For example, a change to a high reinforcement rate in one schedule component typically results in a lower response rate in the other schedule component. A relevant example for the current experiment is when environmental enrichment studies provide concurrent access to alternative reinforcement (e.g. toys and treats) while the drug is 
made readily available for consumption and a decrease in drug use is observed. ${ }^{12-16}$ Likewise, in the current experiment, EE was provided concurrently with ethanol availability that led to a decrease in ethanol consumption by adolescent female rats. Notable to mention is that we observed the treatment effect of $\mathrm{EE}$ while rats were isolated and given concurrent access to ethanol. Previous research has demonstrated that social interaction is an important component of EE on decreasing drug consumption. For example, studies have shown that when rats are housed in groups within the EE condition, they show significantly decreased drug consumption..$^{12}$ Indeed, previous research has demonstrated that social isolation is a stressful condition for rodents and therefore, may lead to an increase in drug consumption. ${ }^{19,26}$ However, the current study's results suggest that EE is still a viable treatment option for reducing ethanol consumption even when placed in the stressed-induced condition of social isolation.

An important aspect of the current study was to examine the changes in adolescent female ethanol consumption after loss of EE stimulation. Previous research has shown that the removal of non-drug alternatives results in the behavioral resurgence of drug responding or an increase in drug taking. ${ }^{18,19}$ Furthermore, in humans, researchers have suggested a link between the removal of alternative, reinforcing events and increases in drug intake or instances of behavioral resurgence after periods of abstinence. ${ }^{25}$ For example, researchers examined data from a Health and Retirement study in order to explore the relationship between involuntary job loss and smoking intensity, as well as, relapse in abstinent smokers. They found that involuntary job loss contributed significantly to elevated levels of smoking in individuals who already smoked. Furthermore, risk of relapse doubled after job loss in ex-smokers. ${ }^{27}$ Similarly, we found that loss of access to non-drug alternatives (EE) led to a significant increase in ethanol consumption for adolescent female rats. Thus, the common feature that both the current and previous studies all demonstrate is that when stimulation or reward is derived from a source other than the drug itself (enrichment), there is a reduction in the reinforcing effects of the drug(s), thereby supporting abstinence. Further, when non-drug alternative rewards are no longer being delivered, a behavioral resurgence of drug use could be the result.

One possible neural mechanism whereby EE may produce its effect on ethanol consumption in adolescent female rats is by disrupting neural circuits in areas involved in ethanol taking. For example, researchers have found that EE housing reduced the incentive value of novelty and the reinforcing properties of ETOH that are mediated by the mesocorticolimbic dopaminergic reward system. ${ }^{28}$ The disruption of neural circuitry by EE is supported by other studies that found EE rats previously trained to self-administer cocaine and after cue-induced relapse tests for cocaine had activated cFos protein in the mesocorticolimbic system to a lesser extent than in NEE animals. ${ }^{7,8,29,30}$ Brain alterations in neural pathways associated with alcohol abuse suggests that EE may play a disruptive role in the neural mechanisms associated with alcohol consumption. Further, this may help explain how the concurrent introduction of rewarding stimulation (EE), may have the effect of reducing the reinforcing effects of ethanol through the contrast mechanism described above.

Previous male animal studies that delivered alternative non-drug rewards such as, food, novelty objects, and voluntary physical activity in the presence of drug found that access to nondrug alternatives led to a decrease in drug-maintained responding.,5-9 Similarly, the current study found that when adolescent female rats were provided access to EE alternatives there was a reduction in ethanol consumption. Therefore, EE may also be an effective treatment strategy for substance abuse when implemented in female populations.

One limitation to the present study is that we did not assess estrous levels in freely cycling adolescent female rats, while in the EE or NEE conditions. Increased estrogen levels have been shown to be related to increased alcohol use in females. ${ }^{31,32}$ However speculative, it may be that EE disrupts the estrogenic effects that contribute to female's increased sensitivity to the rewarding effects of alcohol. In part, this possible explanation could account for significantly less ETOH consumption rates by female rats during the EE condition compared to the NEE condition. Therefore, future studies should examine the possible role that EE may play in mediating the estrogenic effects on alcohol use in adolescent female rats. Another limitation to the present study involves the restricted age cohort of investigating the possible EE preventative effects on ETOH in adolescent female rats. Future studies should examine the heterogeneity of treatment effects concerning EE on ETOH use by observing other female age cohorts.

\section{CONCLUSIONS}

The primary focus of this study was to investigate a potential treatment strategy that would support ETOH abstinence in adolescent female rats. We found that exposure to EE led to significantly less consumption of ETOH for both female groups, while loss of EE led to a significant increase in ethanol volitional intake. The present results support previous research findings (largely with animal male populations) using EE by demonstrating that when having concurrent access to alternative reinforcement (e.g., wheel running) it leads to a reduction in the reinforcing effects of the drug, thereby facilitating abstinence. The current findings may have important implications concerning treatment strategies for alcohol abuse in adolescent females. 


\section{ACKNOWLEDGEMENTS}

The authors thank the State University of New York at Cortland for their generous funding that made this work possible.

\section{REFERENCES}

1. Substance Abuse and Mental Health Services Administration, SAMSHA. National Survey on Drug Use and Health, https:/ / datafiles.sambsa.gov/study-series/national-survey-drug-use-and-health-nsduh-nid13517 (accessed Feb 2018)

2. National Institute on Alcohol Abuse and Alcoholism. Alcohol facts and statistics, http:/ / www.niaaa.nih.gov/alcohol-health/overview-alcohol-consumption/alcohol-facts-and-statistics (accessed Feb 2018)

3. Keyes, K.M., Hatzenbuehler, M.L., Grant, B.F., and Hasin, D.S. (2012) Stress and Alcohol: Epidemiologic Evidence, Alcohol Res: Current Rev 34, 4. https:/ / doi.org/10.1007/s00213-011-2236-1

4. White, A., Castle, I.P., Chen, C.M., Shirley, M., Roach, D., Hingson, R. (2015) Converging 640 Patterns of Alcohol Use and Related Outcomes Among Females and Males in the United 641 States, 2002 to 2012, Alcohol Clinical and Experimental Res., 39, 1712-1726. doi: 10.1111/acer.12815

5. Carroll ME. (1993) The economic context of drug and non-drug reinforcers affects acquisition and maintenance of drug reinforced behavior and withdrawal effects, Drug Alcohol Depend 33, 201-210. bttps:/ / doi.org/ 10.1016/0376-8716(93)90061-T

6. Carroll ME, Lac ST, Nygaard SL. (1989) A concurrently available nondrug reinforcer prevents the acquisition or decreases the maintenance of cocaine reinforced behavior, Psychopharmacol (Berlin) 97, 23-29. doi.org/10.1007/BF004434077

7. Zlebnik NE, Anker JJ, Gliddon LA, Carroll ME. (2010) Reduction of extinction and reinstatement of cocaine seeking by wheel running in female rats, Psychopharmacol 209, 113-125. https:/ / doi.org/10.1007/ s00213-010-1776-0

8. Chauvet C, Lardeux V, Goldberg SR, Jaber M, Solinas M. (2009) Environmental enrichment reduces cocaine seeking and reinstatement induced by cues and stress but not by cocaine, Neuropsychopharmacol 34, 2767-2778. bttps:// doi.org/10.1038/npp.2009.127

9. Thiel KJ, Sanabria F, Pentkowski NS, Neisewander JL. (2009) Anticraving effects of environmental enrichment, Int. J. Neuropsychopharmacol 12, 1151-1156. https:// doi.org/10.1017/S1461145709990472

10. Peck, J. A., Galaj, E., Eshak, S., Newman, K. L., \& Ranaldi, R. (2015) Environmental enrichment induces early heroin abstinence in an animal conflict model, Pharmacol Biochem and Behav 138, 20-25. https://doi.org/10.1016/j.pbb.2015.09.009

11. Ewing, Scott, Ranaldi, Robert. (2018) Environmental enrichment facilitates cocaine abstinence in an animal conflict model, Pharmacol Biochem and Behav 166, 35-41. https:/ / doi.org/10.1016/j.pbb.2018.01.006

12. Solinas M, Thiriet N, Chauvet C, Jaber, M. (2010) Prevention and treatment of drug addiction by environmental enrichment, ScienceDirect 92 (4). https:/ / doi.org/10.1016/j.pneurobio.2010.08.002

13. Lynch WJ, Piehl KB, Acosta G, Peterson AB, Hemby SE. (2010) Aerobic exercise attenuates reinstatement of cocaine-seeking behavior and associated neuroadaptations in the prefrontal cortex, Biol Psychiatry 68, 774-777. bttps:// doi.org/10.1016/j.biopsych.2010.06.022

14. Smith MA, Schmidt KT, Iordanou JC, Mustroph ML. (2008) Aerobic exercise decreases the positive-reinforcing effects of cocaine, Drug Alcohol Depend 98,129-135. https:/ / doi.org/10.1016/j.drugalcdep.2008.05.006

15. Zlebnik NE, Anker JJ, Carroll ME. (2012) Exercise to reduce the escalation of cocaine self-administration in adolescent and adult rats, Psychopharmacol (Berlin) 224, 387-400. https:// doi.org/10.1007/s00213-012-2760-7

16. Cosgrove KP, Hunter RG, Carroll ME. (2002) Wheel-running attenuates intravenous cocaine self-administration in rats: sex differences, Pharmacol Biochem Behav 73, 663-671. https:/ / doi.org/10.1016/S0091-3057(02)00853-5

17. Grimm JW, Osincup D, Wells B, Manaois M, Fyall A. (2008) Environmental enrichment attenuates cue-induced reinstatement of sucrose seeking in rats, Behav Pharmacol 19, 777-785. doi.org/10.1097/FBP.0b013e32831c3b18

18. Podlesnik CA, Jimenez-Gomez C, Shahan TA. (2006) Resurgence of alcohol seeking produced by discontinuing non-drug reinforcement as an animal model of drug relapse, Behav Pharmacol 17, 369-374. doi.org/10.1097/01.fbp.0000224385.09486.ba

19. Nader, Joelle, Claudia, Chauvet, Rawas El Rana, Favot, Laure, Jaber, Mohamed, Thiriet, Nathalie, Solinas, Marcello. (2012) Loss of Environmental Enrichment Increase Vulnerability to Cocaine Addiction, Neuropsychopharmacol 1579-1587. https://doi.org/10.1038/mpp.2012.2

20. Peck, A. Joshua, Galaj, Ewa, Eshak, Stephanie, Newman, L. Kristena, Ranaldi, Robert. (2015) Environmental enrichment induces early heroin abstinence in an animal conflict model, Pharmacol, Biochem Behav 138, 20-25. bttps:/ / doi.org/ 10.1016/j.pbb.2015.09.009

21. Ranaldi R, Kest K, Zellner M, Hachimine-Semperbrom P. (2011) Environmental enrichment, administered after establishment of cocaine self-administration, reduces lever pressing in extinction and during a cocaine context renewal test, Behav Pharmacol 22, 347-353. doi.org/10.1097/FBP.0b013e3283487365

22. Berger, D.F., Lombardo, J.P., Peck, J.A., Faraone, S.V., Middleton, F.A. and Youngetob, S.L. (2010) The effects of strain and prenatal nicotine exposure on ethanol consumption by adolescent male and female rats, Behav Brain Res 210(2), 147-154. bttps://doi.org/10.1016/j.bbr.2010.01.047

23. Reynolds, GS. (1961) Contrast, generalization, and the process of discrimination, J Exp Anal Behav 4, $289-294$. doi.org/10.1901/jeab.1961.4-289 
24. Williams, B.A. (2002) Behavioral contrast redux, Animal learning \& behav 30(1), 1-20. bttps:// doi.org/10.3758/BF03192905

25. Troisi JR II (2013) Perhaps more consideration of Pavlovian-operant interaction may improve the clinical efficacy of behaviorally based drug treatment programs. Psychol Rec 63:863-894. https:/ / doi.org/10.11133/j.tpr.2013.63.4.010

26. Raz, Sivan., Berger, BD. (2010) Social isolation increases morphine intake: behavioral and psychopharmacological aspects. Behav Pharmacol 21(1) 39-46. doi: 10.1097/FBP.0b013e32833470bd

27. Falba T, Teng H, Sindelar JL, Gallo WT. (2005) The effect of involuntary job loss on smoking intensity and relapse, Addiction 100, 1330-1339. https://doi.org/10.1111/j.1360-0443.2005.01150.x

28. de Carvalho, C.R., Pandolfo, P., Pamplona, F.A. and Takahashi, R.N. (2010) Environmental enrichment reduces the impact of novelty and motivational properties of ethanol in spontaneously hypertensive rats, Behav Brain Res 208(1), $231-236$. bttps:// doi.org/10.1016/j.bbr.2009.11.043

29. Chauvet, C., Lardeux, V., Jaber, M. and Solinas, M. (2011) Brain regions associated with the reversal of cocaine conditioned place preference by environmental enrichment, Neurosci 184, 88-96. https:// doi.org/10.1016/j.neuroscience.2011.03.068

30. Thiel, K.J., Pentkowski, N.S., Peartree, N.A., Painter, M.R. and Neisewander, J.L. (2010) Environmental living conditions introduced during forced abstinence alter cocaine-seeking behavior and Fos protein expression, Neurosci 171(4), 11871196. https:// doi.org/10.1016/j.neuroscience.2010.10.001

31. Lenz, B, Müller, C. P., Stoessel, C., Sperling, W., Biermann, T., Hillemacher, T., Bleich, S., Kornhuber, J. (2012) Sex hormone activity in alcohol addiction: integrating organizational and activational effects, Progress in Neurobio 96, 136-163. https:// doi.org/10.1016/j.pneurobio.2011.11.001

32. Hudson, A., Stamp. J. A. (2011) Ovarian hormones and propensity to drug relapse: a review, Neurosci and Biobehav Rev 35, 427-436. https:/ / doi.org/10.1016/j.neubiorev.2010.05.001

\section{ABOUT STUDENT AUTHORS}

Natalie Lipari will graduate in 2020 with a Bachelor of Science degree in Psychology and plans to continue her research focus in the area of substance abuse as she pursues her Ph.D. in Behavioral Neuroscience. Max Baron will graduate in 2021 from the University of Michigan with a Bachelor of Science degree in Biopsychology, Cognition and Neuroscience. He plans to continue research in the area of Behavioral Neuroscience upon graduate school acceptance.

\section{PRESS SUMMARY}

Recent research has shown that female adolescent alcohol abuse is on the rise in the United States. In fact, the number of adolescent females (ages 12-17) with an alcohol use disorder (AUD) actually surpassed the number of adolescent males with an AUD. The consequences of underage drinking can be devastating and may result in truancy, motor vehicle injuries, sexual assault cases, and even death. Thus, with the rise of adolescent female alcohol consumption and increased risk of AUD, it is essential to determine a treatment method that supports long-term alcohol abstinence in females. One potential treatment is the implementation of environmental enrichment (EE). Environmental enrichment is a process concerning the stimulation of the brain by one's physical and social surrounding that promotes non-drug reinforcement alternatives supporting drug abstinence. Further, accumulating evidence indicates that exposure to an EE during the earlier stages of life reduces the effects of abused drugs and decreases the vulnerability to develop a substance abuse disorder. To our knowledge, there have been no studies that have examined EE as a possible treatment strategy to support alcohol abstinence in adolescent female rats. Therefore, the current research sought to examine this novel approach by implementing EE as a viable treatment option to support abstinence in female alcohol abuse. The results suggest that access to enriched life conditions are important in facilitating alcohol abstinence in adolescent female rats. 